OPEN ACCESS

Edited by: Bruno Sainz Jr.

Autonomous University of Madrid, Spain

Reviewed by:

Julie Earl,

Ramón y Cajal Institute for Health

Research, Spain

Gernot Kriegshäuser,

Landes Krankenhaus Steyr, Austria

*Correspondence:

Xiao-Rong Pan xrpan@126.com

Jian-Hua Tong

jh_tong@126.com

Specialty section: This article was submitted to Gastrointestinal Cancers,

a section of the journal

Frontiers in Oncology

Received: 29 July 2019 Accepted: 07 November 2019 Published: 29 November 2019

Citation:

Wang Z-Y, Ding X-Q, Zhu H, Wang R-X, Pan X-R and Tong J-H (2019) KRAS Mutant Allele Fraction in Circulating Cell-Free DNA Correlates With Clinical Stage in Pancreatic

Cancer Patients.

Front. Oncol. 9:1295.

doi: 10.3389/fonc.2019.01295

\section{KRAS Mutant Allele Fraction in Circulating Cell-Free DNA Correlates With Clinical Stage in Pancreatic Cancer Patients}

\author{
Zhe-Ying Wang, Xiao-Qing Ding, Hui Zhu, Rui-Xian Wang, Xiao-Rong Pan* and \\ Jian-Hua Tong*
}

Faculty of Medical Laboratory Science and Central Laboratory, Ruijin Hospital, Shanghai Jiao Tong University School of Medicine, Shanghai, China

Background: The research on circulating tumor DNA (ctDNA) in pancreatic cancer (PC) has emerged recently. Although the detection rate of the KRAS mutation in ctDNA was relatively consistent with that in tumor tissue, whether the KRAS mutant allele fraction (MAF) differed was still not reported. So far, the clinical application of ctDNA detection in $\mathrm{PC}$ remains inconclusive.

Methods: Plasma samples were collected from $110 \mathrm{PC}$ and 52 pancreatic benign (PB) disease patients. The detection of KRAS mutation in ctDNA was performed using droplet digital PCR and compared with that in matched tumor tissue. We assessed the utility of KRAS MAFs in ctDNA and tissue for pancreatic malignancy assessment.

Results: We found that KRAS MAF in ctDNA of PC patients was higher than that of PB patients, and was obviously associated with tumor staging and distant metastasis. However, KRAS MAF in ctDNA was significantly different from that in matched tissue. KRAS MAF in tumor tissue had no significant correlation with the clinical status. In addition, a ROC curve analysis revealed that mutant KRAS ctDNA combined with CA19-9 could increase the sensitivity rate of early-stage PC prediction, compared with CA19-9 test alone.

Conclusion: The MAF of KRAS in ctDNA was related to the clinical stage of PC $(p=0.001)$. Mutant KRAS ctDNA could improve the sensitivity in early diagnosis of PC as a complement to CA19-9. Our study suggested that KRAS mutation in ctDNA could be a valuable circulating biomarker for the malignancy assessment in PC.

Keywords: circulating tumor DNA, KRAS mutation, pancreatic cancer, liquid biopsy, droplet digital PCR

\section{INTRODUCTION}

Pancreatic cancer (PC) is one of the most lethal malignancies in the world, with a 5-year overall survival rate of $<6 \%(1,2)$. The poor prognosis is partly due to the fact that $\mathrm{PC}$ is usually diagnosed at advanced stages and resistant to therapy (3). Therefore, the opportunity for improving patient prognosis lies in earlier diagnosis and monitoring of cancer progress and recurrence. Usually, carbohydrate antigen 19-9 (CA19-9) is a routine tumor biomarker used in the diagnosis and 
management of patients with PC. However, the application of CA19-9 is limited due to its low specificity in non-malignant situations, such as cholestasis or diabetes mellitus $(4,5)$.

As is well-known, the occurrence and the development of cancer results from the accumulation of genetic aberrations in DNA. Therefore, these genetic alterations could serve as specific biomarkers for cancer patients. It is reported that KRAS is one of the most frequently mutated genes in PC, with a detection rate in tumor tissue ranging from 75 to $95 \%$ (6). However, tumor tissue sampling is usually not convenient, particularly for patients who cannot undergo surgical resection. Recently, it has been shown that mutant DNA within tumor cells can be released into blood, and the research on circulating tumor DNA (ctDNA) in plasma has evolved as an exciting field in oncology (7-10). Besides the ease of blood sample acquirement, it is more likely that ctDNA could reflect the overall tumor burden in comparison with tumor tissue. KRAS mutations in ctDNA have been previously studied in the context of PC, with a detection rate ranging from 41.3 to $75 \%(11-14)$. Although the presence of KRAS mutation in ctDNA was relatively consistent with that in tumor tissue of the same person, whether the mutant allele fraction (MAF) of KRAS in ctDNA and tumor tissue differed was still not reported. In addition, since the content of cellfree DNA (cfDNA) in the circulation is too low, the application of ctDNA detection in diagnosis and disease evaluation of PC remains inconclusive. With technologic advances, the methods for quantitative detection of ctDNA, including droplet digital PCR (ddPCR) and optimized next generation sequencing (NGS) strategies, have rapidly developed. Since ddPCR affords a very high level of sensitivity and absolute quantification for the target molecules, it is considered as one of the most important methods for mutant ctDNA detection (15).

In this study, we detected the common hot spot mutations in KRAS of ctDNA using ddPCR and compared it with that of tissue samples using standard clinical testing. In addition, we analyzed the utility of KRAS mutation in ctDNA and paired tissue samples of PC and pancreatic benign disease (PB) patients for malignancy assessment.

\section{MATERIALS AND METHODS}

\section{Plasma Samples}

Whole blood samples from $110 \mathrm{PC}$ and $52 \mathrm{~PB}$ patients were obtained prior to treatment in Ruijin Hospital (Shanghai, China). One to four milliliter of peripheral blood was collected in EDTA tubes $(\mathrm{BD})$ and centrifuged at $1,900 \times \mathrm{g}$ for $10 \mathrm{~min}$ at room temperature and then at $16,000 \times \mathrm{g}$ for $10 \mathrm{~min}$ at $4^{\circ} \mathrm{C}$. Isolated plasma samples were stored at $-80^{\circ} \mathrm{C}$ until needed.

\section{Cell Culture}

Cell lines (PANC-1, BxPC-3, and HCT116) were obtained from Shanghai Institute of Digestive Surgery (Shanghai, China). Briefly, PANC-1, BxPC-3, and HCT116 cells were cultured in DMEM, RPMI 1640, and McCoy's 5A (Gibco) with 10\% fetal bovine serum (Sigma), respectively. Cell culture was performed at $37^{\circ} \mathrm{C}$ in a $5 \% \mathrm{CO}_{2}$ atmosphere. Before supernatant cfDNA isolation, cells were grown at an initial density of 2.5 $\times 10^{5} / \mathrm{ml}$ for $48 \mathrm{~h}$. Then the cell culture supernatants were collected and cleared by two-step centrifugation as described for plasma samples.

\section{cfDNA Isolation and Quantification}

Total cfDNA was extracted from plasma or cell culture supernatant with use of the QIAamp ccfDNA/RNA Kit (Qiagen) according to the manufacturer's protocol, then quantified using the Qubit 3.0 fluorometer (Thermo Fisher). For patient samples, the concentration of cfDNA was indicated as nanograms per milliliter of plasma.

\section{Primers and Probes}

The primers (Beijing Genomics Institute) used to amplify a segment in exon 2 of KRAS gene (78-bp amplicon) were as follows: Forward primer, 5'-GCCTGCTGAAAATGACTGAAT3'; Reverse primer, 5'-GCTGTATCGTCAAGGCACTCT-3'. Multiple hotspot mutations within codon 12 and codon 13 of KRAS gene exon 2 were detected with a pair of drop-off and reference probes according to the method described by Decraene et al. (16). A drop-off probe with a $5^{\prime}$ fluorophore and a $3^{\prime}$ non-fluorescent quencher (NFQ) was designed covering hotspot mutation regions in exon 2 of the wild-type KRAS gene. A reference probe was designed upstream of the drop-off probe over a non-mutated region in the same amplicon, with a 5' fluorophore and a $3^{\prime}$ NFQ. The sequences of these two probes (Life Technologies) were $5^{\prime}$-(6-FAM)-CTACGCCACCAGCT(MGB NFQ)-3' and $5^{\prime}$-(VIC)-CAACTACCACAAGTTT-(MGB NFQ)-3', respectively (Supplementary Figure 1A). Thus, wildtype KRAS molecules were double fluorescence signal positive, while mutant molecules sub-optimally hybridized to the drop-off probe due to a mismatch and presented a decrease in the drop-off probe signal.

\section{Detection of KRAS Mutations in cfDNA}

The ddPCR platform (Qx200 ddPCR system, Bio-Rad) was used for the detection of KRAS mutations in cfDNA as per the manufacturer's instruction. Twenty microliter ddPCR reaction solutions were prepared with dUTP-free Supermix for probes (Bio-Rad), $900 \mathrm{nM}$ of primers, $250 \mathrm{nM}$ of hydrolysis probes and at least $0.5 \mathrm{ng}$ of cfDNA. The amplification was performed under the following conditions: $95^{\circ} \mathrm{C}$ for $10 \mathrm{~min}, 40$ cycles of $\left(94^{\circ} \mathrm{C}\right.$ for $30 \mathrm{~s}, 60^{\circ} \mathrm{C}$ for $\left.60 \mathrm{~s}\right), 98^{\circ} \mathrm{C}$ for $10 \mathrm{~min}$. The results were analyzed by the Quanta-Soft Analysis Pro software (Bio-Rad).

\section{Calculation of the LOB and LOD}

The limit of blank (LOB) and the limit of detection (LOD) for the ddPCR analysis were measured according to the Clinical and Laboratory Standards Institute guidelines EP17.2 and relevant reports (16-18). CfDNA templates extracted from BxPC-3 cells with wild-type KRAS were used as blank samples. Forty replicates of blank samples were detected by the ddPCR assay to calculate the LOB, which corresponds to the 95th percentile of distribution of the blank values. For LOD, cfDNA templates extracted from PANC-1 cells with mutant KRAS (c.35G>A, p.G12D) and HCT116 cells with mutant KRAS (c.38G>A, p.G13D) were diluted with the wild-type cfDNA to the expected MAFs $(5,2.5$, 
$1.25,0.63,0.31,0.16$, and $0.08 \%$ ), respectively. Then a series of cfDNA samples with different MAFs were detected by the ddPCR assay and repeated for at least 6 independent experiments. After consideration on the results of G12D and G13D, the LOD was defined as the MAF value in which the $95 \%$ confidence interval of all replicate detections presented values above the LOB.

TABLE 1 | Patient characteristics.

\begin{tabular}{|c|c|c|c|}
\hline \multicolumn{2}{|c|}{ Groups } & $\begin{array}{l}\text { Pancreatic } \\
\text { cancer }\end{array}$ & $\begin{array}{c}\text { Pancreatic } \\
\text { benign disease }\end{array}$ \\
\hline \multicolumn{2}{|c|}{ Patient number (n) } & 110 & 52 \\
\hline \multicolumn{2}{|c|}{ Age, mean (range) (year) } & 65 (40-91) & $55(22-81)$ \\
\hline \multirow{2}{*}{$\begin{array}{l}\text { Gender } \\
\text { (percentage) }\end{array}$} & Female & $47(42.7 \%)$ & 27 (51.9\%) \\
\hline & Male & $63(57.3 \%)$ & $25(48.1 \%)$ \\
\hline \multirow{4}{*}{$\begin{array}{l}\text { Clinical stage } \\
\text { (percentage) }\end{array}$} & 1 & $31(27.4 \%)$ & - \\
\hline & $\|$ & 24 (21.2\%) & - \\
\hline & III & $32(29.1 \%)$ & - \\
\hline & IV & $20(18.2 \%)$ & - \\
\hline \multicolumn{2}{|c|}{ CA19-9, median (range) (U/ml) } & $\begin{array}{c}175.2 \\
(0.8-20190.0)\end{array}$ & $\begin{array}{c}9.0 \\
(0.80-317.40)\end{array}$ \\
\hline \multicolumn{2}{|c|}{ cfDNA, median (range) (ng/ml plasma) } & $\begin{array}{c}8.38 \\
(0.55-95.40)\end{array}$ & $\begin{array}{c}7.83 \\
(0.62-63.93)\end{array}$ \\
\hline
\end{tabular}

cfDNA, cell-free DNA.

\section{Detection of KRAS Status in Tissue Samples}

Matched formalin fixed paraffin embedded (FFPE) tissue specimens were obtained according to the clinical histopathologic results. DNA was extracted using Qiagen GeneRead DNA FFPE Kit (Qiagen) as per the instruction of the manufacturer. The KRAS status was determined using NGS on Illumina MiSeq System (Illumina).

\section{Statistical Analyses}

Statistical analyses were performed using the IBM SPSS Version 23 software (IBM Corporation, Armonk, NY, USA). Continuous data were compared using the Wilcoxon and Kruskal-Wallis tests. The correlation analysis was performed using the Spearman rank test. The accuracy of the circulating biomarkers was analyzed using a receiver operating characteristic (ROC) curve. A $p<0.05$ was considered as significant.

\section{RESULTS}

\section{Patient Characteristics}

In total, 162 patients with pancreatic space occupying lesions (110 malignant and 52 benign) were included in this study. The characteristics of the patients are listed in Table 1. The PC patients included 99 pancreatic ductal adenocarcinoma and 11 other malignant tumors. For the PB patients, the most common diseases diagnosed were serous cystadenoma ( $n=14,26.9 \%)$, pseudocyst ( $n=14,26.9 \%$ ), intraductal papillary mucinoma
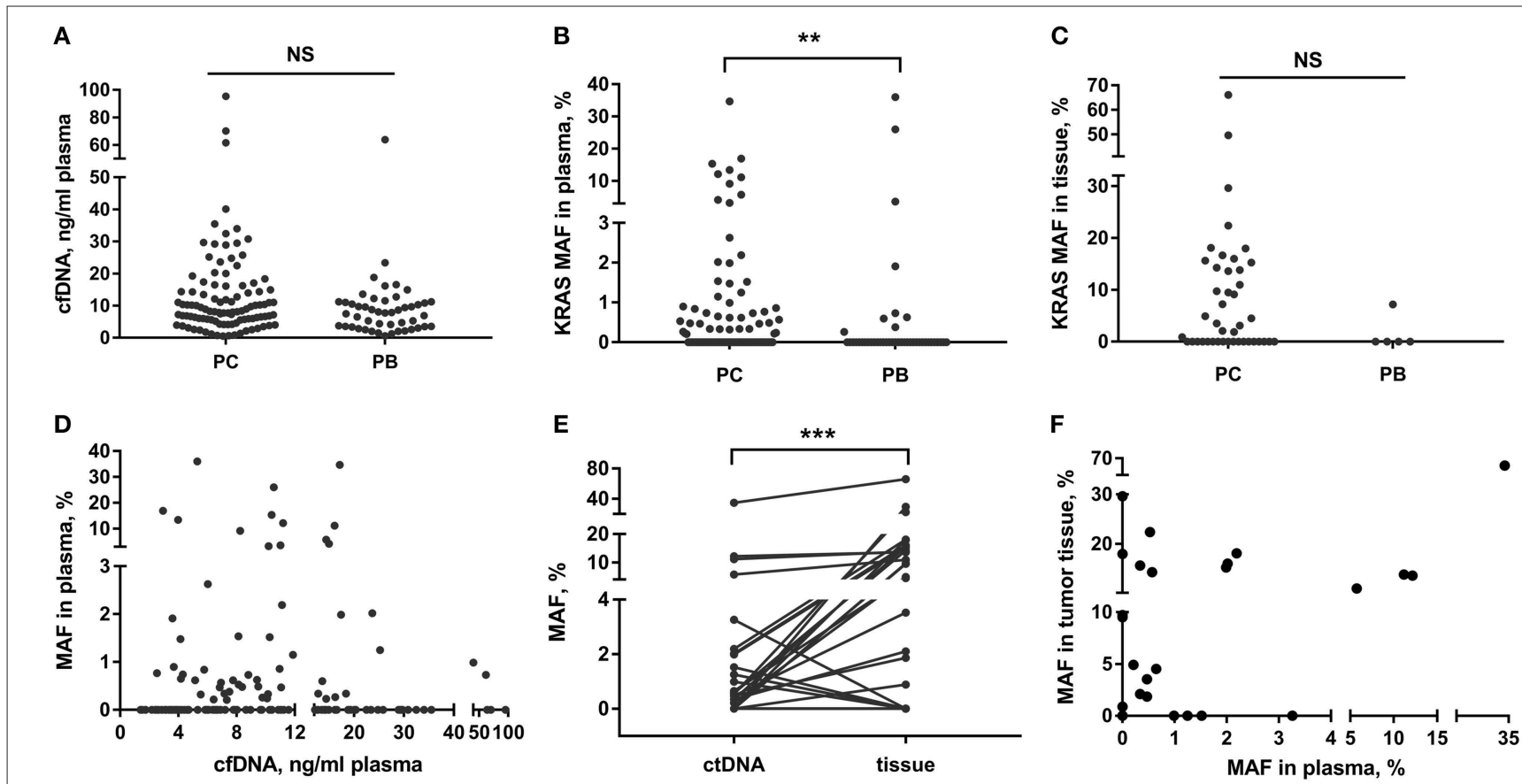

FIGURE 1 | cfDNA content and KRAS mutation in PC and PB patients. (A-C) Detection of total cfDNA amount and KRAS MAF in ctDNA and tissue in PC and PB diseases. (D) Correlation between $K R A S$ MAF in ctDNA and cfDNA concentration. (E,F) Comparison of $K R A S$ MAF in ctDNA and matched tissue. ${ }^{\star \star} P<0.01$; ${ }^{\star * \star} p<0.001 ;$ NS, not significant. 
( $n=11,21.2 \%)$, and mucinous cystadenoma $(n=9,17.3 \%)$. The median CA19-9 concentration in PC was higher than that in PB (175.2 U/ml vs. $9.0 \mathrm{U} / \mathrm{ml}, p<0.001)$.

\section{KRAS Mutation Status in cfDNA and Pancreatic Tissue}

In this study, we detected that the LOB of the ddPCR analysis on the mutant KRAS ctDNA was $0.10 \%$. The LODs were $0.16 \%$ for 2 $\mathrm{ng}$ and $5 \mathrm{ng}$ of cfDNA templates, $0.63 \%$ for $1 \mathrm{ng}$, and $1.25 \%$ for 0.5 ng (Supplementary Figures 1B,C). The cfDNA content did not show obvious differences in the PC and PB groups (Figure 1A, $p=0.066)$. Then the ddPCR detection was performed in 138 (85\%) cases with adequate cfDNA amount in the study cohort. The detection rate of mutant KRAS ctDNA were $47.4 \%$ (45/95) in $\mathrm{PC}$ and $20.9 \%$ (9/43) in PB, respectively. KRAS mutation in tissue was present in $58.1 \%(25 / 43)$ of malignant samples and $20 \%$ (1/5) of benign samples, respectively (Figure 1C). KRAS MAFs in ctDNA of PC were much higher than that of $\mathrm{PB}$ (Figure 1B, $p$ $=0.007$ ). In addition, we demonstrated there was no significant correlation between cfDNA concentration and KRAS MAF in ctDNA (Figure 1D, $p=0.663, r^{2}=0.001$ ).

\section{Discordance of MAFs Between ctDNA and Matched Tissue}

We also compared the KRAS mutation status in ctDNA and matched tissue in 35 patients (34 PC and $1 \mathrm{~PB}$ ). The blood and tissue samples were collected at the same time. The concordance rate of KRAS mutation status (mutant or wild-type) in ctDNA and tissue was $74.3 \%(26 / 35)$ (Table 2$)$. However, the MAF of $K R A S$ in ctDNA was significantly different from that in tumor tissue of the same person (Figure 1E, $p<0.001$ ). The median MAFs of KRAS in ctDNA and tumor tissue were $0.34 \%$ (range: $0-$ $34.71 \%$ ) and $2.81 \%$ (range: $0-66.10 \%$ ), respectively. In addition, the correlation between KRAS MAFs in ctDNA and tumor tissue was poor (Figure 1F, $p=0.007, r^{2}=0.200$ ).

\section{KRAS MAF in ctDNA in Relation to Clinical Stages of PC}

We then analyzed the correlation between KRAS MAF in ctDNA or tumor tissue and clinical characteristics of PC patients. The $K R A S$ MAF in ctDNA was obviously associated with clinical staging $(p=0.001)$ and the presence of distant metastasis $(p$ $<0.001$ ) in PC (Table 3, Figure 2A). Patients in stage IV had significantly greater KRAS MAF in ctDNA than patients in stage

TABLE 2 | Results of KRAS mutation detection in ctDNA and matched tissue.

\begin{tabular}{llccc}
\hline \multirow{2}{*}{ Groups } & \multicolumn{3}{c}{ PC or PB tissue } \\
\cline { 3 - 5 } & & KRAS mutant & KRAS wild-type & Total \\
\hline \multirow{2}{*}{ ctDNA } & KRAS mutant & 15 & 4 & 19 \\
& KRAS wild-type & 5 & 11 & 16 \\
\hline & Total & 20 & 15 & 35 \\
\hline
\end{tabular}

ctDNA, circulating tumor DNA.
I/II $(p<0.001$ and 0.031). KRAS MAFs in patients of stage I and II were all below $2 \%$. In comparison with KRAS MAF in ctDNA, there was no statistically significant difference in KRAS MAF in tumor tissue, when the PC patients were stratified with either clinical staging or the presence of distant metastasis (Table 3, Figure $2 B, p=0.498$ and 0.370 ).

\section{KRAS Mutation in ctDNA and Tissue as Biomarkers of Malignancy Prediction}

Furtherly, we analyzed the diagnostic utility of KRAS mutation in ctDNA, CA19-9, and KRAS mutation in tissue as PC biomarkers (Table 4). ROC curve analysis showed that the overall sensitivity and specificity of mutant KRAS ctDNA detection were 47 and $80 \%$, respectively. According to the cancer stages, the sensitivity rates of the ctDNA detection were $30,46,40$, and $83 \%$, respectively (Figure 2C). In order to improve the sensitivity of the circulating biomarker test, the combination of mutant KRAS ctDNA with CA19-9 was employed. In comparison with mutant KRAS ctDNA detection or CA19-9 test alone, the overall sensitivity of the combined assay was increased to $82 \%$ at the specificity of $81 \%$ (Table 4 ). The sensitivity rates of the combined assay in cancer stage I-IV were improved to $82,82,83$, and $89 \%$, respectively (Figure 2C).

\section{DISCUSSION}

In recent years, ctDNA has gained substantial attention in the field of clinical oncology (19). The mutations of KRAS were detected in numerous tumors. In PC, the presence of KRAS mutation in ctDNA and tumor tissue was relatively consistent and the concordance rate was around 54-91\% (20-24). However, the comparison between the MAF of KRAS in ctDNA and tumor tissue was rarely reported. In this study, we compared the detection rate of KRAS mutation in ctDNA with that in pancreatic tissue and found their concordance rate was $74.3 \%$. Furthermore, we analyzed the MAF of KRAS in PC, and revealed for the first time that the KRAS MAF in ctDNA was significantly different from that in matched tumor tissue. In contrast to KRAS MAF in ctDNA, KRAS MAF in tumor tissue was not obviously different in PC patients stratified with either tumor staging or distant metastasis. Some studies also reported that the prognostic utility of KRAS mutation in ctDNA and in tumor tissue was discordant. Mutant KRAS ctDNA was considered to be a better prognostic biomarker for PC, whereas KRAS MAF in tumor tissue was not $(14,25)$. These findings might be due to the fact that a part of tumor tissue could only provide limited information. As we know, tumor tissue is very heterogeneous and will inevitably be contaminated with non-tumor cells that will skew the MAF data results $(24,26)$. Partial tissue sampling may lead to false negative results of mutation detection. However, ctDNA could better reflect the overall tumor burden in PC patients. The detection of ctDNA could improve the mutation detection rate as a good supplement to tumor tissue.

Current studies on PC showed that the total amount of circulating cfDNA and KRAS mutations in ctDNA might be associated with pancreatic tumor burden, but the conclusions 
TABLE 3 | Correlation of KRAS MAFs with clinical status of pancreatic cancer patients.

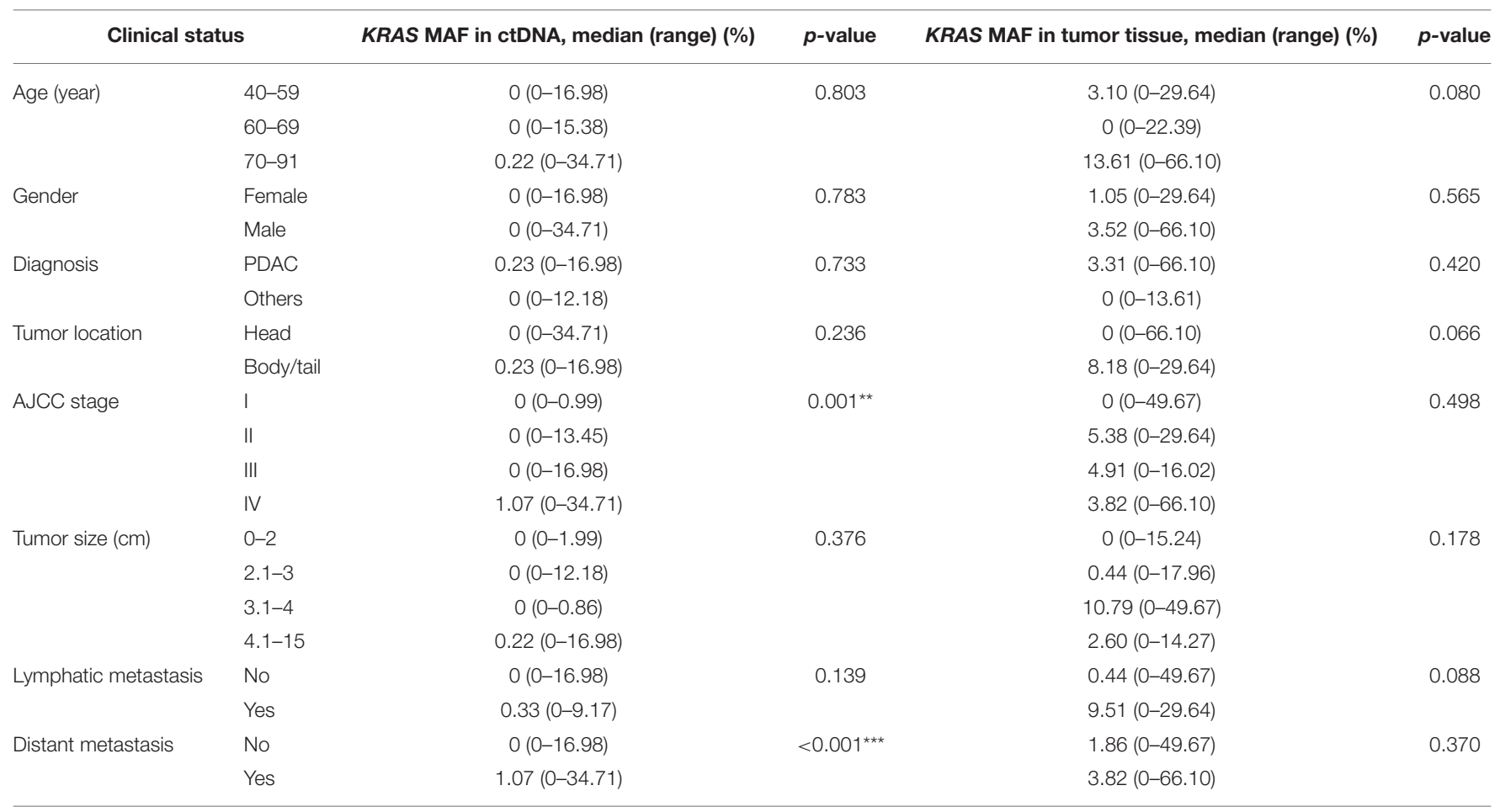

MAF, mutant allele fraction; ctDNA, circulating tumor DNA; PDAC, pancreatic ductal adenocarcinoma; AJCC, American Joint Committee on Cancer. ${ }^{* *} p<0.01,{ }^{* \star *} p<0.001$.
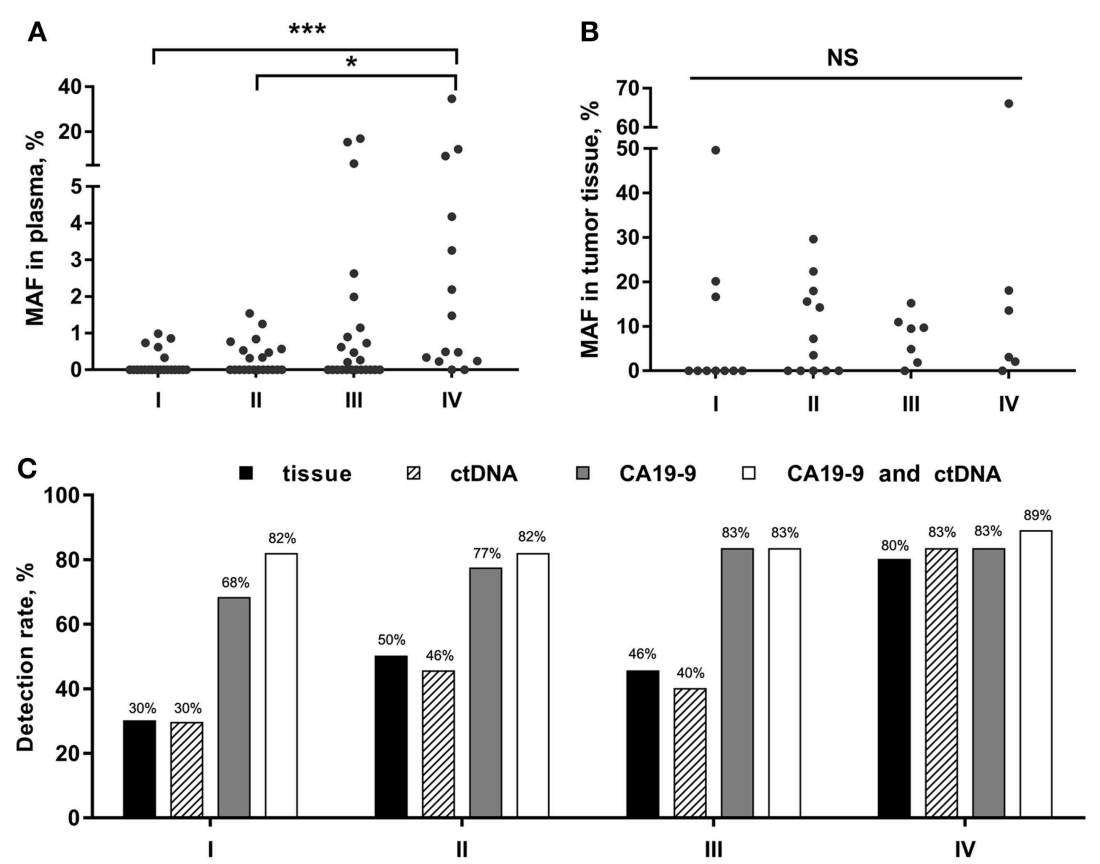

FIGURE 2 | KRAS MAFs and the detection rate for PC in different clinical stages. (A,B) Correlation of KRAS MAF in ctDNA and tumor tissue with PC stages. (C) PC detection rate by tissue, ctDNA, CA19-9, and the combined assay of ctDNA and CA19-9. ${ }^{*} p<0.05$; ${ }^{\star \star *} p<0.001$; NS, not significant.

remained inconsistent. For example, Wei et al. demonstrated that the total cfDNA level of patients with advanced PC was higher than that of healthy controls. The PC patients in stage
IV had greater KRAS MAFs in ctDNA than patients in stage III (27). Bernard et al. found that the KRAS MAFs in ctDNA were significantly different in patients with pancreatic cysts, 
TABLE 4 | Performance of circulating biomarkers in comparison with KRAS mutation in tissue for malignancy prediction.

\begin{tabular}{lccc}
\hline Tests & $\begin{array}{c}\text { Patient } \\
\text { number }(\boldsymbol{n})\end{array}$ & Sensitivity (\%) & Specificity (\%) \\
\hline $\begin{array}{l}\text { KRAS MAF in } \\
\text { PB or PC tissue }\end{array}$ & 43 & 42 & 100 \\
KRAS MAF in ctDNA & 93 & 47 & 80 \\
CA19-9 & 100 & 76 & 85 \\
$\begin{array}{l}\text { Combination assay of } \\
\text { ctDNA and CA19-9 }\end{array}$ & 93 & 82 & 81 \\
\hline
\end{tabular}

MAF, mutant allele fraction; ctDNA, circulating tumor DNA.

localized PC, and metastatic PC (20). On the other hand, some studies reported that mutant KRAS ctDNA or total cfDNA were statistically irrelevant with respect to tumor staging (28, 29). In the present study, we detected that PC patients had higher levels of KRAS MAF in ctDNA in comparison with $\mathrm{PB}$ patients. Moreover, KRAS MAF in ctDNA was obviously associated with tumor staging and distant metastasis in PC patients. PC patients in stage IV had greater KRAS MAF in ctDNA than patients in stage I/II $(p<0.001$ and 0.031$)$, while there was no significant difference between KRAS MAFs in ctDNA in stage III and stage IV patients $(p=0.209)$. Our results showed that KRAS MAF in ctDNA might be a good indicator of tumor burden in PC. In addition, unlike the mutant ctDNA, the total cfDNA concentration showed no significant difference in PB and different stages of PC patients. Similarly, there was no statistically significant correlation between total cfDNA concentration and KRAS MAF in ctDNA. Of note, we also found that the mutant KRAS ctDNA was detected in $20.9 \%(9 / 43)$ of $\mathrm{PB}$ patients. According to the literature, genetic mutations such as KRAS mutations may occur in healthy people and patients with benign diseases $(30,31)$. For these people, whether these genetic mutations were pre-cancerous or normal events needs to be explored through large-scale and long-term experiments.

Though the KRAS mutation in ctDNA was presumed to be a promising diagnostic biomarker of PC, it could only be detected in nearly half of PC patients, with detection rates of $24.4-34 \%$ in early stage patients and $53-74 \%$ in advanced patients $(12,20,29)$. Therefore, the application of mutant KRAS ctDNA as a predictor of PC was limited because of its relatively low sensitivity. CA19-9 is routinely detected in the diagnosis and management of patients with PC. However, the well-known limitation of CA19-9 is its low specificity in non-malignant situations such as cholestasis (32). The combined assay of mutant ctDNA and protein biomarkers such as CA19-9 was performed for PC diagnosis in several studies, showing higher sensitivity than mutant ctDNA detection alone (33-35). In this study, we analyzed the sensitivity rates of KRAS mutation in ctDNA and tumor tissue for PC prediction and found that neither was satisfactory. In the combined assay of mutant KRAS ctDNA and CA19-9, the sensitivity for PC diagnosis was obviously improved especially in the early cancer stages. Compared with each single test, the combined sensitivity was raised to $82 \%$ from $30 \%$ (ctDNA) and 68\% (CA19-9) in stage
I and $82 \%$ from $46 \%$ (ctDNA) and 77\% (CA19-9) in stage II, respectively. Therefore, mutant KRAS ctDNA might be helpful in early diagnosis of PC as a complement to CA19-9.

In order to better apply these study results in clinical practice, it is essential to establish a standardization to compare the data obtained from different investigators (36). The LOD is the value that can be detected in samples with minimum analytes, which is used to reflect the sensitivity of the detection. In theory, the quantity of DNA templates in PCR assay will influence the LOD value. According to our data, we showed that the LOD value of the ddPCR detection increased while the amount of cfDNA templates decreased. Thus, when the LOD value of the detection was provided, the amount of DNA template used should be indicated. Only MAF value above the corresponding LOD could be defined as positive result.

In conclusion, our study showed that KRAS MAF in ctDNA differed from that in tumor tissue in PC. The mutant KRAS ctDNA was found to be related with the clinical stage of PC patients, whereas KRAS MAF in tumor tissue was not. In addition, the combination of mutant KRAS ctDNA and CA199 could be a valuable circulating biomarker for early detection and diagnosis of PC. Nevertheless, here we only carried out a study on PC patients before treatment. The dynamic change of KRAS MAF in ctDNA of PC patients during treatment should be continuously detected in our next work. The clinical application of mutant KRAS ctDNA in the diagnosis and monitoring of PC still needs further validation by large-scale clinical studies.

\section{DATA AVAILABILITY STATEMENT}

The datasets generated for this study are available on request to the corresponding author.

\section{ETHICS STATEMENT}

The studies involving human participants were reviewed and approved by Ruijin Hospital Ethics Committee. The patients/participants provided their written informed consent to participate in this study.

\section{AUTHOR CONTRIBUTIONS}

Z-YW and J-HT designed the study. Z-YW, X-QD, HZ, and R-XW conducted experiments, analyzed data, and wrote the manuscript. Z-YW, X-RP, and J-HT supervised research, interpreted data, and revised the manuscript.

\section{FUNDING}

This work was supported in part by National Natural Science Foundation of China (81470317 and 81170508), Leading Talent Program of Shanghai Municipal Human Resources, and Social Security Bureau. 


\section{ACKNOWLEDGMENTS}

We thank the Clinical Laboratory of Ruijin Hospital, Shanghai Jiao Tong University School of Medicine for the technical support.

\section{REFERENCES}

1. Long J, Luo GP, Xiao ZW, Liu ZQ, Guo M, Liu L, et al. Cancer statistics: current diagnosis and treatment of pancreatic cancer in Shanghai, China. Cancer Lett. (2014) 346:273-7. doi: 10.1016/j.canlet.2014.01.004

2. Vincent A, Herman J, Schulick R, Hruban RH, Goggins M. Pancreatic cancer. Lancet. (2011) 378:607-20. doi: 10.1016/S0140-6736(10)62307-0

3. Hezel AF, Kimmelman AC, Stanger BZ, Bardeesy N, Depinho RA. Genetics and biology of pancreatic ductal adenocarcinoma. Genes Dev. (2006) 20:121849. doi: $10.1101 / \operatorname{gad} .1415606$

4. Goonetilleke KS, Siriwardena AK. Systematic review of carbohydrate antigen (CA 19-9) as a biochemical marker in the diagnosis of pancreatic cancer. Eur J Surg Oncol. (2007) 33:266-70. doi: 10.1016/j.ejso.2006.10.004

5. Ni XG, Bai XF, Mao YL, Shao YF, Wu JX, Shan Y, et al. The clinical value of serum CEA, CA19-9, and CA242 in the diagnosis and prognosis of pancreatic cancer. Eur J Surg Oncol. (2005) 31:164-9. doi: 10.1016/j.ejso.2004.09.007

6. Forbes SA, Beare D, Boutselakis H, Bamford S, Bindal N, Tate J, et al. COSMIC: somatic cancer genetics at high-resolution. Nucleic Acids Res. (2017) 45:D77783. doi: 10.1093/nar/gkw1121

7. Garlan F, Laurent-Puig P, Sefrioui D, Siauve N, Didelot A, Sarafan-Vasseur $\mathrm{N}$, et al. Early evaluation of circulating tumor DNA as marker of therapeutic efficacy in metastatic colorectal cancer patients (PLACOL Study). Clin Cancer Res. (2017) 23:5416-25. doi: 10.1158/1078-0432.CCR-16-3155

8. Garrigou S, Perkins G, Garlan F, Normand C, Didelot A, Le Corre $\mathrm{D}$, et al. A study of hypermethylated circulating tumor DNA as a universal colorectal cancer biomarker. Clin Chem. (2016) 62:112939. doi: 10.1373/clinchem.2015.253609

9. Haselmann V, Gebhardt C, Brechtel I, Duda A, Czerwinski C, Sucker A, et al. Liquid profiling of circulating tumor DNA in plasma of melanoma patients for companion diagnostics and monitoring of BRAF inhibitor therapy. Clin Chem. (2018) 64:830-42. doi: 10.1373/clinchem.2017.281543

10. Oxnard GR, Paweletz CP, Kuang Y, Mach SL, O'Connell A, Messineo $\mathrm{MM}$, et al. Noninvasive detection of response and resistance in EGFR-mutant lung cancer using quantitative next-generation genotyping of cell-free plasma DNA. Clin Cancer Res. (2014) 20:1698-705. doi: 10.1158/1078-0432.CCR-13-2482

11. Cheng H, Liu C, Jiang J, Luo G, Lu Y, Jin K, et al. Analysis of ctDNA to predict prognosis and monitor treatment responses in metastatic pancreatic cancer patients. Int J Cancer. (2017) 140:2344-50. doi: 10.1002/ijc.30650

12. Uemura T, Hibi K, Kaneko T, Takeda S, Inoue S, Okochi O, et al. Detection of K-ras mutations in the plasma DNA of pancreatic cancer patients. $J$ Gastroenterol. (2004) 39:56-60. doi: 10.1007/s00535-003-1245-1

13. Tjensvoll K, Lapin M, Buhl T, Oltedal S, Steen-Ottosen Berry K, Gilje $\mathrm{B}$, et al. Clinical relevance of circulating KRAS mutated DNA in plasma from patients with advanced pancreatic cancer. Mol Oncol. (2016) 10:63543. doi: 10.1016/j.molonc.2015.11.012

14. Pietrasz D, Pecuchet N, Garlan F, Didelot A, Dubreuil O, Doat S, et al. Plasma circulating tumor DNA in pancreatic cancer patients is a prognostic marker. Clin Cancer Res. (2017) 23:116-23. doi: 10.1158/1078-0432.CCR-16-0806

15. Olmedillas-Lopez S, Garcia-Arranz M, Garcia-Olmo D. Current and emerging applications of droplet digital PCR in oncology. Mol Diagn Ther. (2017) 21:493-510. doi: 10.1007/s40291-017-0278-8

16. Decraene C, Silveira AB, Bidard FC, Vallee A, Michel M, Melaabi S, et al. Multiple hotspot mutations scanning by single droplet digital PCR. Clin Chem. (2018) 64:317-28. doi: 10.1373/clinchem.2017.272518

17. Felix-Urquidez D, Perez-Urquiza M, Valdez Torres JB, Leon-Felix J, GarciaEstrada R, Acatzi-Silva A. Development, optimization, and evaluation of a duplex droplet digital PCR assay to quantify the T-nos/hmg copy

\section{SUPPLEMENTARY MATERIAL}

The Supplementary Material for this article can be found online at: https://www.frontiersin.org/articles/10.3389/fonc. 2019.01295/full\#supplementary-material

number ratio in genetically modified maize. Anal Chem. (2016) 88:8129. doi: 10.1021/acs.analchem.5b03238

18. Lund HL, Hughesman CB, Fakhfakh K, McNeil K, Clemens S, Hocken K, et al. Initial diagnosis of ALK-positive non-small-cell lung cancer based on analysis of ALK status utilizing droplet digital PCR. Anal Chem. (2016) 88:4879-85. doi: 10.1021/acs.analchem.6b00707

19. Qi ZH, Xu HX, Zhang SR, Xu JZ, Li S, Gao HL, et al. The significance of liquid biopsy in pancreatic cancer. J Cancer. (2018) 9:341726. doi: 10.7150/jca.24591

20. Bernard V, Kim DU, San Lucas FA, Castillo J, Allenson K, Mulu FC, et al. Circulating nucleic acids are associated with outcomes of patients with pancreatic cancer. Gastroenterology. (2019) 156:108-18 e4. doi: 10.1053/j.gastro.2018.09.022

21. Kinugasa H, Nouso K, Miyahara K, Morimoto Y, Dohi C, Tsutsumi K, et al. Detection of K-ras gene mutation by liquid biopsy in patients with pancreatic cancer. Cancer. (2015) 121:2271-80. doi: 10.1002/cncr.29364

22. Kruger S, Heinemann V, Ross C, Diehl F, Nagel D, Ormanns S, et al. Repeated mutKRAS ctDNA measurements represent a novel and promising tool for early response prediction and therapy monitoring in advanced pancreatic cancer. Ann Oncol. (2018) 29:2348-55. doi: 10.1093/annonc/mdy417

23. Lee B, Lipton L, Cohen J, Tie J, Javed AA, Li L, et al. Circulating tumor DNA as a potential marker of adjuvant chemotherapy benefit following surgery for localized pancreatic cancer. Ann Oncol. (2019) 30:14728. doi: 10.1093/annonc/mdz200

24. Guo Q, Wang J, Xiao J, Wang L, Hu X, Yu W, et al. Heterogeneous mutation pattern in tumor tissue and circulating tumor DNA warrants parallel NGS panel testing. Mol Cancer. (2018) 17:131. doi: 10.1186/s12943-018-0875-0

25. Haas M, Ormanns S, Baechmann S, Remold A, Kruger S, Westphalen CB, et al. Extended RAS analysis and correlation with overall survival in advanced pancreatic cancer. Br J Cancer. (2017) 116:1462-9. doi: 10.1038/bjc.2017.115

26. Keller L, Pantel K. Unravelling tumour heterogeneity by single-cell profiling of circulating tumour cells. Nat Rev Cancer. (2019) 19:55367. doi: 10.1038/s41568-019-0180-2

27. Wei T, Zhang Q, Li X, Su W, Li G, Ma T, et al. Monitoring tumor burden in response to FOLFIRINOX chemotherapy via profiling circulating cell-free DNA in pancreatic cancer. Mol Cancer Ther. (2019) 18:196203. doi: 10.1158/1535-7163.MCT-17-1298

28. Del Re M, Vivaldi C, Rofi E, Vasile E, Miccoli M, Caparello C, et al. Early changes in plasma DNA levels of mutant KRAS as a sensitive marker of response to chemotherapy in pancreatic cancer. Sci Rep. (2017) 7:7931. doi: 10.1038/s41598-017-08297-z

29. Nakano Y, Kitago M, Matsuda S, Nakamura Y, Fujita Y, Imai S, et al. KRAS mutations in cell-free DNA from preoperative and postoperative sera as a pancreatic cancer marker: a retrospective study. Br J Cancer. (2018) 118:6629. doi: 10.1038/bjc.2017.479

30. Yang S, Che SP, Kurywchak P, Tavormina JL, Gansmo LB, Correa de Sampaio P, et al. Detection of mutant KRAS and TP53 DNA in circulating exosomes from healthy individuals and patients with pancreatic cancer. Cancer Biol Ther. (2017) 18:158-65. doi: 10.1080/15384047.2017.1281499

31. Alborelli I, Generali D, Jermann P, Cappelletti MR, Ferrero G, Scaggiante $B$, et al. Cell-free DNA analysis in healthy individuals by next-generation sequencing: a proof of concept and technical validation study. Cell Death Dis. (2019) 10:534. doi: 10.1038/s41419-019-1770-3

32. Su SB, Qin SY, Chen W, Luo W, Jiang HX. Carbohydrate antigen 19-9 for differential diagnosis of pancreatic carcinoma and chronic pancreatitis. World J Gastroenterol. (2015) 21:4323-33. doi: 10.3748/wjg.v21.i14.4323

33. Cohen JD, Javed AA, Thoburn C, Wong F, Tie J, Gibbs P, et al. Combined circulating tumor DNA and protein biomarker-based liquid biopsy for the 
earlier detection of pancreatic cancers. Proc Natl Acad Sci USA. (2017) 114:10202-7. doi: 10.1073/pnas.1704961114

34. Dianxu F, Shengdao Z, Tianquan H, Yu J, Ruoqing L, Zurong Y, et al. A prospective study of detection of pancreatic carcinoma by combined plasma K-ras mutations and serum CA19-9 analysis. Pancreas. (2002) 25:336-41. doi: 10.1097/00006676-20021100000003

35. Sefrioui D, Blanchard F, Toure E, Basile P, Beaussire L, Dolfus C, et al. Diagnostic value of CA19.9, circulating tumour DNA and circulating tumour cells in patients with solid pancreatic tumours. $B r \quad J$ Cancer. (2017) 117:1017-25. doi: 10.1038/bjc.2 017.250

36. Perez-Gracia JL, Sanmamed MF, Bosch A, Patino-Garcia A, Schalper KA, Segura V, et al. Strategies to design clinical studies toidentify predictive biomarkers in cancer research. Cancer Treat Rev. (2017) 53:7997. doi: $10.1016 /$ j.ctrv.2016.12.005

Conflict of Interest: The authors declare that the research was conducted in the absence of any commercial or financial relationships that could be construed as a potential conflict of interest.

Copyright (c) 2019 Wang, Ding, Zhu, Wang, Pan and Tong. This is an open-access article distributed under the terms of the Creative Commons Attribution License (CC $B Y)$. The use, distribution or reproduction in other forums is permitted, provided the original author(s) and the copyright owner(s) are credited and that the original publication in this journal is cited, in accordance with accepted academic practice. No use, distribution or reproduction is permitted which does not comply with these terms. 